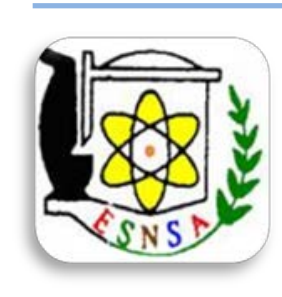

Arab Journal of Nuclear Sciences and Applications

ISSN 1110-0451

Web site: ajnsa.journals.ekb.eq

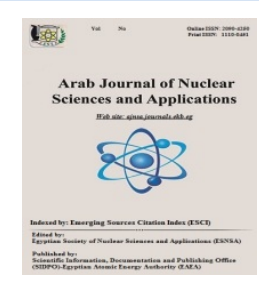

(ESNSA)

\title{
Production of Lightest Neutral MSSM Higgs Boson in Association with Neutralinos at Electron Positron Colliders
}

\author{
Ibrahim S. Mahmoud ${ }^{1}$, T. A. El-Azim² \\ ${ }^{(1)}$ Department of Physics, Faculty of Science, Suez Canal University, Ismailia, Egypt \\ ${ }^{(2)}$ Department of Physics, Faculty of Science, Cairo University, Giza, Egypt \\ E-mail: ${ }^{(1)}$ ibrahim_mahmoud@science.suez.edu.eg
}

\begin{abstract}
Received $16^{\text {th }}$ Apr 2017 This investigation deals with the results of a calculation of the cross section for the production of the Accepted $30^{\text {th }}$ May 2017 lightest Higgs boson in association with an electron and a neutralino at electron-positron colliders $\left(e^{-} e^{+} \rightarrow h \widetilde{\chi}_{i}^{0} \bar{\chi}_{j}^{0}\right)$, where $i, j=1-4$, and $\widetilde{\chi}_{i}^{0}$ donates Neutralinos particles, in the framework of the minimal supersymmetric standard model (MSSM). The basic features of the MSSM prediction for some distinctive parameter scenarios were studied. The associated production of light neutral Higgs bosons and a pair of neutralinos at electron-positron colliders at different production's modes have been carefully examined. It was found that the dominant production can reach a few $\mathrm{fb}$, in the allowed ranges of parameters.
\end{abstract}

Keywords: Light Higgs boson, Neutralinos, minimal supersymmetric standard model, Supersymmetry, Total cross section, MSSM scenarios

\section{Introduction}

The search for the Higgs particles is one of the most challenging problems of experimental particle physics. The theoretical framework is the Minimal Supersymmetric Standard Model[1-3], which, as compared to the Standard Model (SM)[4-6], has an extended scalar sector with two doublets of Higgs fields. In this sector there are two important parameters namely, the Higgs doublet mixing angle, $\alpha$, and the ratio of the doublet vacuum expectation values, $\tan \beta$.

The two-doublets of Higgs fields lead to five physical Higgs bosons, among which three are neutral. The other two particles are the charged Higgs bosons, denoted $H^{ \pm}$. In CP-conserving (CPC) MSSM models, two of the three neutral Higgs bosons, denoted h, for the lighter one, and
$\mathrm{H}$, for the heavier one, are CP-even scalars. The third one is a CP-odd pseudo-scalar, denoted A.

The lighter CP-even scalar $h$, is expected to be relatively light $m_{h} \leq 130-140 \mathrm{GeV}$ [7,8], and its discovery could be the first signal of Supersymmetry.

The Higgs mechanism could be studied in great detail at future $e \bar{e}$ colliders[9,10] at LHC. The upper limit of the light Higgs mass is found to be $m \_h<186$ at the 95\% confidence level (CL)[1114].

One major task of a future $e \bar{e}$ colliders will be the exploration of the Higgs sector[10,15,16] in the Standard Model and beyond it. It is, therefore, interesting to attempt to study the different modes

Corresponding Author: ibrahim_mahmoud@science.suez.edu.eg

DOI: 10.21608 /ajnsa.2018.6509

(C)Scientific Information, Documentation and Publishing Office (SIDPO)-EAEA 
of production of the light Higgs boson, h, in those colliders, especially in the MSSM model, which is considered as the main focus of this paper.

The present work is an extension to a previous study by the authors [17] and is devoted to discuss the production of light Higgs processes represented by the equation: $e^{-} e^{+} \rightarrow h \tilde{\chi}_{i}^{0} \overline{\tilde{\chi}}_{j}^{0}$

The outline of this paper is as follows: section 2 is devoted to giving the MSSM Scenarios definitions. Then, in section 3 , the different modes of production processes of the interaction are defined. The results of the production mechanism are given in section 4. Finally, the conclusions of this work are presented in section 5 .

\section{MSSM Benchmark Scenarios}

In the unconstrained version of the MSSM, no particular Supersymmetry breaking mechanism is assumed, but rather a parameterization of all possible soft SUSY breaking terms is used. This leads to more than a hundred parameters (masses, mixing angles, phases) in this model in addition to the ones of the Standard Model. While a detailed scanning over the more-than-hundred dimensional parameter space of the MSSM is clearly not practicable, even a sampling of a three or fourdimensional parameter space of SUSY is beyond the present capabilities for phenomenological studies, in particular when it comes to simulating experimental signatures within the detectors. For this reason one often resorts to specific benchmark scenarios, i.e. one studies only specific parameter points or at best samples a one-dimensional parameter space (the latter is sometimes called a model line $[18,19])$, which exhibit specific characteristics of the MSSM parameter space. Benchmark scenarios of this kind are often used, for instance, to study the performance of different experiments at the same collider. Similarly, detailed experimental simulations of sparticle production with identical MSSM parameters in the framework of different colliders can be very helpful in developing strategies for combining pieces of information obtained at different machines.

\section{MSSM model parameters}

The production of neutral Higgs bosons is tested in MSSM with seven parameters. Two of these parameters are sufficient to describe the Higgs sector at tree level. As a convenient choice, $\tan \beta$ and (the mass of the CP-odd Higgs boson) $m_{A}$ are chosen in the case of the CP-Conservation scenario. There are additional parameters appear at the level of radiative corrections:

$M_{\text {Susy }}, M_{2}, \mu, A$, and $m_{\tilde{g}}$. All soft SUSY-breaking parameters in the sfermion sector are set to $M_{\text {Susy }}$ at the electroweak scale. $M_{2}$ is the $\mathrm{SU}(2)$ gaugino mass parameter at the electroweak scale and $M_{1}$, the $\mathrm{U}(1)$ gaugino mass parameter, is derived from $M_{2}$ using the GUT relation $M_{1}=M_{2}\left(\frac{5 \sin ^{2} \theta_{w}}{3 \cos ^{2} \theta_{w}}\right)$, where $\theta_{w}$ is the weak mixing angle. The supersymmetric Higgs mass parameter is denoted $\mu$. The parameter $A=A_{t}=A_{b}$ is the common trilinear Higgs-squark coupling for up-type and down-type squarks. The stop and sbottom mixing parameters are defined as $X_{t}=A_{t}-\cot \beta$ and $X_{b}=A_{b}-\tan \beta$. The parameter $m_{\tilde{g}}$ is the gluino mass. Large radiative corrections to the predicted mass $m_{h}$ arise from scalar top loops, while the contributions from scalar bottom loops are smaller. The precise mass of the top quark has a strong impact on $m_{h}$; it is taken to be $m_{t}=174.3 \mathrm{GeV}$, the current average of the Tevatron measurements[20].

The question of which parameter choices are useful as benchmark scenarios depends on the purpose of the actual investigation. The LEP2 collaborations have performed analysis for the MSSM model using several benchmark scenarios $[13,21,22]$ that were considered as typical cases for MSSM parameters space. Among those different benchmark sets, we investigate one type, the $\left(m_{h}^{\max }\right)$ benchmark Scenario.

The maximal mixing $\left(m_{h}^{\max }\right)$ benchmark Scenario If one is interested, for instance, in setting exclusion limits on the SUSY parameter space from the non-observation of SUSY signals at the experiments performed up to now, it is useful to use a benchmark scenario which gives rise to conservative exclusion bounds. An example of a benchmark scenario of this kind is the maximal mixing set $\left(m_{h}^{\max }\right) \backslash$ cite[23]. It gives rise to maximal values of the lightest CP-even Higgsboson mass (for fixed values of the top-quark mass and the SUSY scale) and thus allows one to set conservative bounds on $\tan \beta$ and $m_{A}$. In this scenario the stop mixing parameter is set to a large value, $X_{t}=\sqrt{6} \mathrm{M}_{\text {Susy }}$, in the $\overline{M S}$ renormalization 
scheme, and $X_{t}=2 M_{\text {Susy }}$ in the $\overline{F D}$ renormalization scheme. This scenario is designed to maximize the theoretical upper bound of $m_{h}$ for a given value of $\tan \beta$ and fixed values of $m_{t}$ and $\mathrm{M}_{\text {Susy }}$. This model thus provides a wider parameter space and therefore more conservative exclusion limits than the other scenarios. The values of $\mu$ and $M_{2}$ are close to their experimental lower bounds. Slightly higher Higgs-boson masses are obtained for smaller $|\mu|$ and smaller $M_{2}$. The sign of $\mu$ has only a small effect in this scenario. The parameters values of this scenario are shown in Table (I)

\section{The Production of Lightest Higgs Boson with Neutralinos}

Kinematics

We study the reaction

$$
\boldsymbol{e}^{-}\left(\boldsymbol{p}_{\mathbf{1}}\right) \boldsymbol{e}^{+}\left(\boldsymbol{p}_{\mathbf{2}}\right) \rightarrow \boldsymbol{h}(\boldsymbol{k}) \tilde{\chi}_{i}^{0}\left(\boldsymbol{p}_{3}\right) \overline{\tilde{\chi}}_{j}^{0}\left(\boldsymbol{p}_{\mathbf{4}}\right)
$$

Where $\overline{\tilde{\chi}}_{i}^{0}$ donates any Neutralinos particles in MSSM model that could participate in the reaction, $i, j=1-4$, and $p_{1}$ and $p_{2}$ denote the momenta of initial-states of electron and positron particles, $k, p_{3}$, and $p_{4}$ are the momenta of the final-state of lightest higgs $(\boldsymbol{h})$ and the associated Neutralinos particles $\left(\tilde{\chi}_{i}^{0}, \overline{\tilde{\chi}}_{j}^{0}\right)$.

In addition, the electron mass is neglected, and unpolarized electron and positron beams are assumed. Thus, the differential cross section is summed over spin polarization of the final-states. The production cross section is evaluated by numerical integration over the kinematically allowed range.

\section{Feynman diagrams}

The Feynman diagrams for each sub-process of the interaction can be classified into the five channels shown in Fig. 1. Each channel represents an interaction process path, from the incident $e^{-} e^{+}$particles states toward the final ones, which includes the light Higgs (h), through different propagators $\left(P_{r_{1}} \& P_{r_{2}}\right)$ according to the rules of MSSM model.

\section{The subprocesses of interaction}

The production of the lightest Higgs at the $e^{-} e^{+}$ colliders in a three-body final state process is carried out through a definite number of subprocesses. In each sub-process, the associated pairs of sleptons and the lightest Higgs boson, produced in the final state follows the Feynman rules in the MSSM model.

\section{Grouping of Sub-processes}

By careful study of the distributions of Feynman diagrams depending on the channels distribution of Fig. 1, one could rearrange the subprocesses into two groups. By studying each group, it could be estimated which subprocess in the group has the main role in the production. In the following subsections those groups are determined and carefully studied.

Group I. This group includes the sub-processes number (1), (5), (8), and (10). It may be represented by the following interaction: $e^{-} e^{+} \rightarrow$ $h \widetilde{\chi}_{i}^{0} \overline{\widetilde{\chi}}_{i}^{\mathbf{0}}$, where $i=1-4$, the associated external particles are the Neutralinos-antineutralinos pairs.

Group II. This group includes the sub-processes numbers $(2,3,4),(6,7)$, and (9). The produced Neutralino-antineutralino pairs are dissimilar. It may represented by the following interaction: $e^{-} e^{+} \rightarrow \boldsymbol{h} \widetilde{\chi}_{i}^{\mathbf{0}} \overline{\widetilde{\chi}}_{\boldsymbol{j}}^{\mathbf{0}}$, where $i, j=1-$ $4, i \neq j$.

\section{Numerical Calculations}

Firstly, the calculations begin by identifying the different allowed subprocesses of the interaction in the framework of the MSSM model in Feynman gauge. After that, the Feynman diagrams of each allowed sub-process is obtained, and then the squared matrix element of each sub-process is calculated. The production cross section is generated with the Calchep code [24].

For the purpose of our analysis, we take as independent MSSM parameters: the ratio of the neutral Higgs VEV's $\tan \beta$, and higgsino mass parameter $\mu$. Three values of the parameter $\tan \beta$ (= $10,20,30$ ) have been chosen to study the production process. The choice of $\mu$ parameter is determined by scanning over the values $\mu(=-200$, $200,400)$ for a certain sub-process in each group. The production cross section scan process results are shown in Fig. 2. From the results of this scan, the $\mu$ parameter was fixed at a value of $-200 \mathrm{GeV}$, since it maximizes the value of the cross section. 
For instance, this choice of parameters yields the particles spectrum shown in table (2).

Table (I): The maximal mixing $\left(\mathrm{m}_{\mathrm{h}}^{\max }\right)$ benchmark Scenario parameters

\begin{tabular}{llllllll}
\hline & & & & & & \\
& & $M_{\text {Susy }}$ & $m_{t}$ & $m_{2}$ & $m_{\widetilde{g}}$ & $\mu$ & $X_{t}$ \\
& & & & & & & \\
$\begin{array}{l}\text { Value } \\
\text { (in GeV) }\end{array}$ & 1000 & 174.3 & 200 & $\begin{array}{l}0.8 \\
M_{\text {Susy }}\end{array}$ & -200 & $\sqrt{6} M_{\text {Sus }}$
\end{tabular}

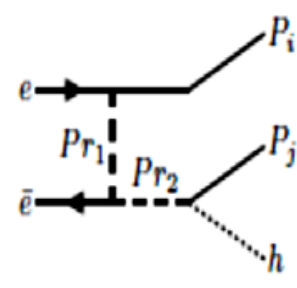

(a)

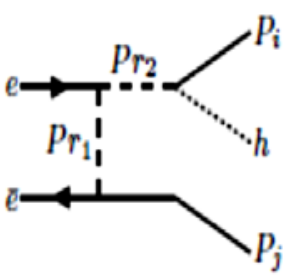

(b)

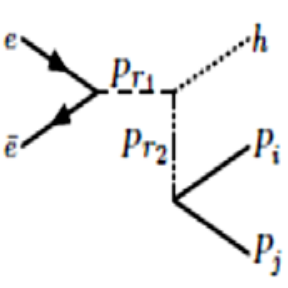

(c)

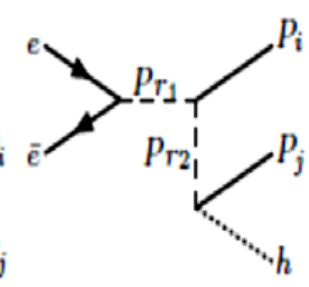

(d)

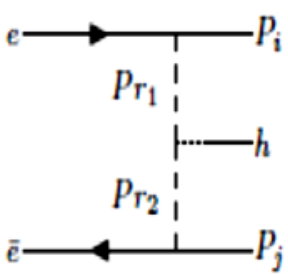

(e)

Fig. (1): The channels classification of Feynman diagrams in the different sub-processes of the interaction. $P_{i} \& P_{j}$ are the Neutralinos particles, where $P_{r_{1}} \& P_{r_{2}}$ are propagators

Table (II): The sub-processes of the Interaction $\mathbf{e}^{-} \mathbf{e}^{+} \rightarrow \mathbf{h} \tilde{\chi}_{\mathbf{i}}^{0} \overline{\tilde{\chi}}_{\mathbf{j}}^{0}$

\begin{tabular}{|c|c|c|c|c|c|}
\hline z & 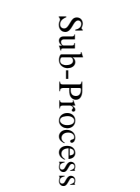 & 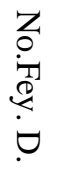 & z & $\begin{array}{l}\mathscr{N} \\
\tilde{J} \\
\underline{1} \\
0 \\
0 \\
0 \\
\infty \\
心\end{array}$ & $\begin{array}{l}Z \\
0 \\
i_{1} \\
\text { T) } \\
\dot{0} \\
\dot{0}\end{array}$ \\
\hline (1) & $\begin{array}{l}\mathrm{e}^{-} \mathrm{e}^{+} \\
\rightarrow \mathrm{h} \tilde{\chi}_{4}^{0} \overline{\tilde{X}}_{4}^{0}\end{array}$ & 24 & (2) & $\begin{array}{l}\mathrm{e}^{-} \mathrm{e}^{+} \\
\rightarrow \mathrm{h} \tilde{\chi}_{3}^{0} \bar{\chi}_{4}^{0}\end{array}$ & 46 \\
\hline (3) & $\begin{array}{l}\mathrm{e}^{-} \mathrm{e}^{+} \\
\rightarrow \mathrm{h} \tilde{\chi}_{2}^{0} \overline{\tilde{\chi}}_{4}^{0}\end{array}$ & 46 & (4) & $\begin{array}{l}\mathrm{e}^{-} \mathrm{e}^{+} \\
\rightarrow \mathrm{h} \tilde{\chi}_{1}^{0} \overline{\tilde{\chi}}_{4}^{0}\end{array}$ & 46 \\
\hline (5) & $\begin{array}{l}\mathrm{e}^{-} \mathrm{e}^{+} \\
\rightarrow \mathrm{h} \tilde{\chi}_{3}^{0} \bar{\chi}_{3}^{0}\end{array}$ & 24 & (6) & $\begin{array}{l}\mathrm{e}^{-} \mathrm{e}^{+} \\
\rightarrow \mathrm{h} \tilde{\chi}_{2}^{0} \bar{\chi}_{3}^{0}\end{array}$ & 46 \\
\hline (7) & $\begin{array}{l}\mathrm{e}^{-} \mathrm{e}^{+} \\
\rightarrow \mathrm{h} \tilde{\chi}_{1}^{0} \bar{\chi}_{3}^{0}\end{array}$ & 46 & (8) & $\begin{array}{l}\mathrm{e}^{-} \mathrm{e}^{+} \\
\rightarrow \mathrm{h} \tilde{\chi}_{2}^{0} \bar{\chi}_{2}^{0}\end{array}$ & 24 \\
\hline (9) & $\begin{array}{l}\mathrm{e}^{-} \mathrm{e}^{+} \\
\rightarrow \mathrm{h} \tilde{\chi}_{1}^{0} \bar{\chi}_{2}^{0}\end{array}$ & 46 & (10) & $\begin{array}{l}\mathrm{e}^{-} \mathrm{e}^{+} \\
\rightarrow \mathrm{h} \tilde{\chi}_{1}^{0} \bar{\chi}_{1}^{0}\end{array}$ & 24 \\
\hline
\end{tabular}



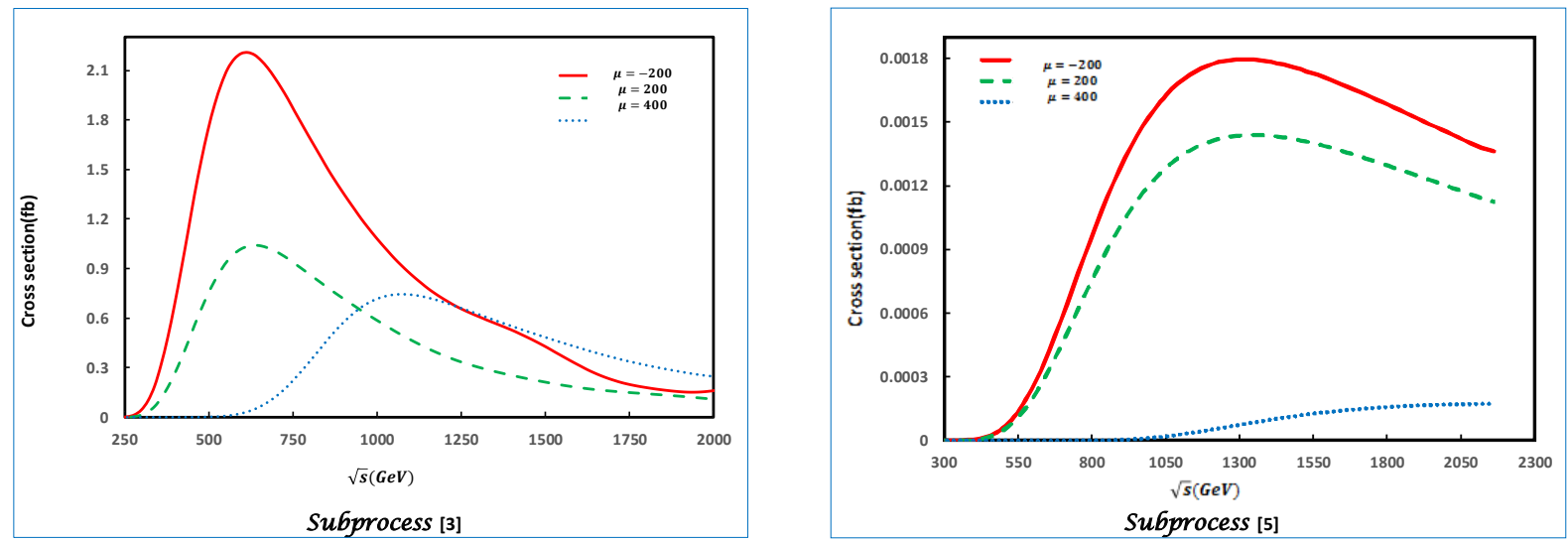

Fig. (2): The production cross sections $(\sigma)$ as a function of the cm energy $(\sqrt{s})$ at $\mu=[-200$ (red - ), 200 (green - - -), and 400 (blue $\cdots .$.$) ]$

\section{Results}

The production cross sections $(\sigma)$ in unit of (fb) for the associated production of the lightest neutral Higgs particles with a pairs of sleptons are shown as a function of the $\mathrm{cm}$. energy $(\sqrt{s})$ for interactions at three values of the parameter $\tan \beta(=10,20$, 30) at $\mu=-200 \mathrm{GeV}$.

\section{Group I}

Fig. 3 shows the total cross section for the subprocesses of group (I). Table 3 concludes the maximum values of production cross section and its corresponding $\mathrm{cm}$. energy for this group. Using the previous table we conclude that the sub-process 9 has the dominant contribution in the production mechanism.

\section{Group II}

Fig. 4 shows the total cross section for the subprocesses of group (II). Table 4 lists the maximum values of production cross section and its corresponding $\mathrm{cm}$. energy for this group. From this table we notice that the sub-process 2 has the dominant contribution in the production mechanism.

\section{Reaction Summery}

Table (V):II summarizes the dominant sub-process for each group. From this table we notice that the most promising sub-process is $9\left(\mathrm{e}^{-} \mathrm{e}^{+} \rightarrow \mathrm{h} \tilde{\chi}_{1}^{0} \overline{\tilde{\chi}}_{2}^{0}\right)$, which has the output particles LSP (Least Supersymmetric Particle $\tilde{\chi}_{1}^{0}$ ) in associated with second Neutralino, and higgs boson, and accordingly the most suitable value for the $\tan \beta$ parameter is 10

\section{Conclusions}

In this work, the production of the lightest neutral Higgs bosons in association with pairs of neutralinos in the MSSM model at electronpositron collider has been studied at three different values of both $\mu$ and $\tan \beta$ parameters in the framework of MSSM maximal-mixing benchmark scenarios. The reaction proceeds from input to output final states through ten sub-processes. For the sub-process $\mathrm{e}^{-} \mathrm{e}^{+} \rightarrow \mathrm{h} \tilde{\chi}_{1}^{0} \overline{\tilde{\chi}}_{2}^{0}$ of group (II) at $\sqrt{\mathrm{s}}=379 \mathrm{GeV}, \mu=-200$, and $\tan \beta=10$, the production cross-section is found to be the most promising and dominant process which has the largest production cross section among the others $(\sigma=6.934 f b)$. On the other hand, the subprocess $\mathrm{e}^{-} \mathrm{e}^{+} \rightarrow \mathrm{h} \tilde{\chi}_{3}^{0} \overline{\tilde{\chi}}_{3}^{0}$ of group (I) at $\sqrt{\mathrm{s}}=$ $1328 \mathrm{GeV}, \quad \tan \beta=30$, and $\mu=-200$, the production cross-section is found to be the least possible process, which has the lowest value for the cross-section $\left(\sigma=1.706 \times 10^{-3} \mathrm{fb}\right)$.

Therefore, one could conclude that the optimal parameters value for the production of lightest MSSM Higgs in $\mathrm{e}^{-} \mathrm{e}^{+}$colliders at maximal benchmark are $\tan \beta=10$ and $\mu=-200$ at c.m energy $(\sqrt{s} \simeq 400 \mathrm{GeV})$. The noticed signature of this reaction might be the production of second neutralino and a missing energy stands for the production of the first neutralino, which consider as the LSP. 
Table (III): The production cross-section and $\mathrm{cm}$. energy for subprocesses of group I at $\mu=-200$

\begin{tabular}{|c|c|c|}
\hline \multirow{2}{*}{$\begin{array}{c}\text { SubPrs. } \\
\text { NO. }\end{array}$} & \multicolumn{2}{|c|}{$\tan \beta=10$} \\
\hline & $\sqrt{\mathrm{s}}(\mathrm{GeV})$ & $\sigma(f b)$ \\
\hline [1] & 1310 & $5.100 \times 10^{-3}$ \\
\hline [5] & 1310 & $1.795 \times 10^{-3}$ \\
\hline [8] & 419 & 4.836 \\
\hline [10] & 340 & 2.375 \\
\hline \multirow{2}{*}{$\begin{array}{c}\text { SubPrs. } \\
\text { NO. }\end{array}$} & \multicolumn{2}{|c|}{$\tan \beta=20$} \\
\hline & $\sqrt{\mathrm{s}}(\mathrm{GeV})$ & $\sigma(f b)$ \\
\hline [1] & 1320 & $5.439 \times 10^{-3}$ \\
\hline [5] & 1329 & $1.728 \times 10^{-3}$ \\
\hline [8] & 439 & 4.387 \\
\hline [10] & 340 & 2.446 \\
\hline \multirow{2}{*}{$\begin{array}{c}\text { SubPrs. } \\
\text { NO. }\end{array}$} & \multicolumn{2}{|c|}{$\tan \beta=30$} \\
\hline & $\sqrt{\mathrm{s}}(\mathrm{GeV})$ & $\sigma(f b)$ \\
\hline [1] & 1289 & $5.559 \times 10^{-3}$ \\
\hline [5] & 1328 & $1.706 \times 10^{-3}$ \\
\hline [8] & 457 & 3.778 \\
\hline [10] & 398 & 2.159 \\
\hline
\end{tabular}
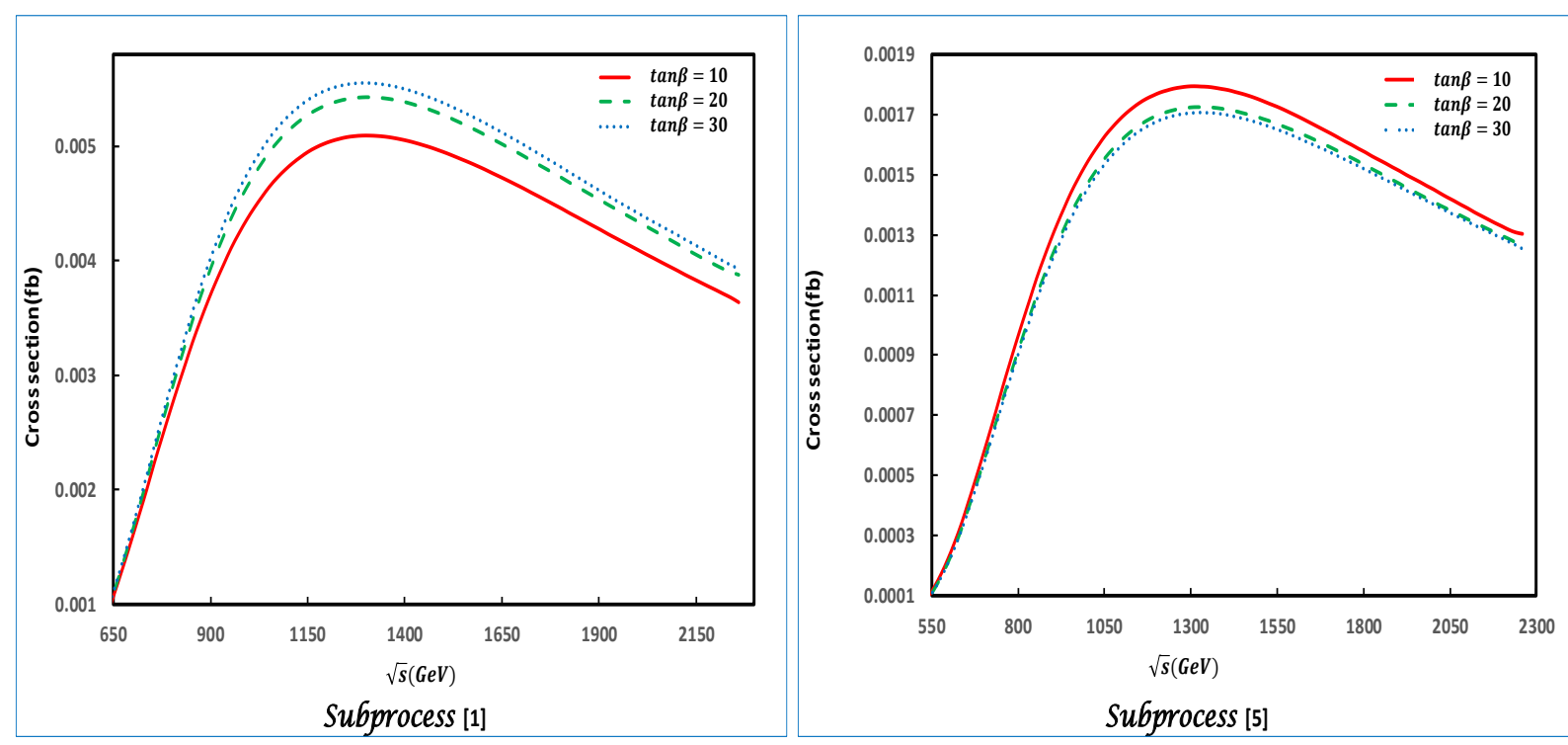


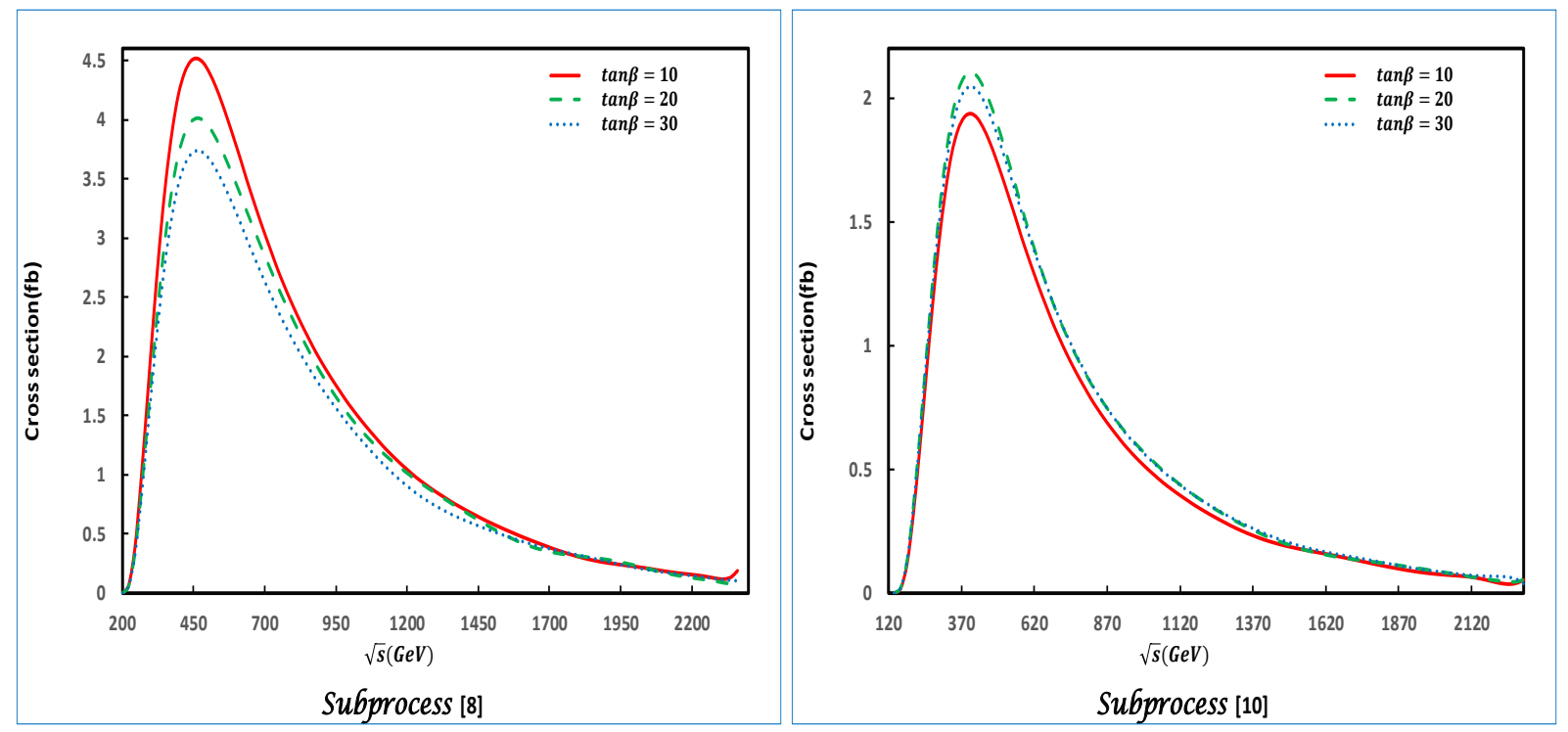

Fig. (3): The production cross sections $(\sigma)$ as a function of the cm. energy $(\sqrt{s})$ for group I at $\tan \beta=[10$ (red -$), 20$ (green - - ), and 30 (blue $\cdots$...) ] at $\mu=-200 \mathrm{GeV}$.

Table (IV): The production cross-section and $\mathrm{cm}$. energy for subprocesses of group II at $\boldsymbol{\mu}=\mathbf{2 0 0}$

\begin{tabular}{|c|c|c|}
\hline \multirow{2}{*}{ SubPrs. NO. } & \multicolumn{2}{|c|}{$\tan \beta=10$} \\
\hline & $\sqrt{\mathrm{s}}(\mathrm{GeV})$ & $\sigma(f b)$ \\
\hline [2] & 814 & $6.757 \times 10^{-3}$ \\
\hline [3] & 597 & 2.298 \\
\hline [4] & 597 & 1.910 \\
\hline [5] & 597 & 2.042 \\
\hline [7] & 597 & 5.020 \\
\hline [9] & 379 & 6.934 \\
\hline \multirow{2}{*}{ SubPrs. NO. } & \multicolumn{2}{|c|}{$\tan \beta=20$} \\
\hline & $\sqrt{\mathrm{s}}(\mathrm{GeV})$ & $\sigma(f b)$ \\
\hline [2] & 814 & $6.908 \times 10^{-3}$ \\
\hline [3] & 597 & 1.806 \\
\hline [4] & 597 & 1.924 \\
\hline [5] & 597 & 4.727 \\
\hline [7] & 597 & 2.267 \\
\hline [9] & 379 & 6.648 \\
\hline \multirow{2}{*}{ SubPrs. NO. } & \multicolumn{2}{|c|}{$\tan \beta=30$} \\
\hline & $\sqrt{\mathrm{s}}(\mathrm{GeV})$ & $\sigma(f b)$ \\
\hline [2] & 834 & $6.935 \times 10^{-3}$ \\
\hline [3] & 616 & 1.569 \\
\hline [4] & 616 & 1.724 \\
\hline [5] & 616 & 4.164 \\
\hline [7] & 616 & 2.236 \\
\hline [9] & 418 & 5.681 \\
\hline
\end{tabular}



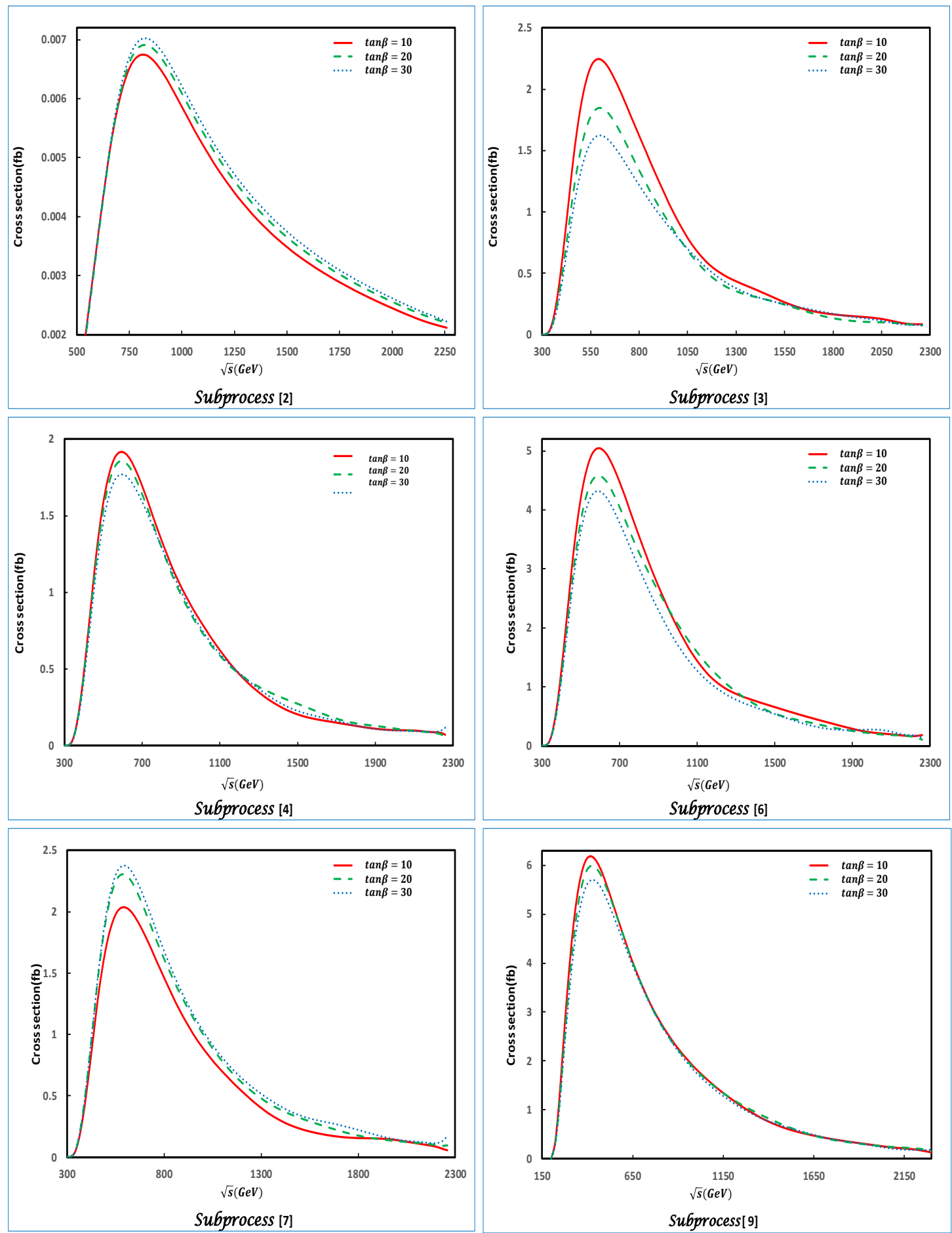

Fig. (4): The production cross sections $(\sigma)$ as a function of the $\mathrm{cm}$. energy $(\sqrt{s})$ for group II at $\tan \beta=[10$ (red -), 20 (green - - ), and 30 (blue $\cdots$. ) ] at $\mu=-200 \mathrm{GeV}$. 
Table (V):II The summarize of the dominant subprocesses

\begin{tabular}{|c|c|c|}
\hline \multirow{2}{*}{$\begin{array}{l}\text { SubPrs. } \\
\text { NO. }\end{array}$} & \multicolumn{2}{|c|}{$\tan \beta=10$} \\
\hline & $\sqrt{\mathrm{s}}(\mathrm{GeV})$ & $\sigma(f b)$ \\
\hline$[\mathrm{I}-8]$ & 419 & 4.836 \\
\hline [II-9] & 379 & 6.934 \\
\hline \multirow{2}{*}{$\begin{array}{l}\text { SubPrs. } \\
\text { NO. }\end{array}$} & \multicolumn{2}{|c|}{$\tan \beta=20$} \\
\hline & $\sqrt{\mathrm{s}}(\mathrm{GeV})$ & $\sigma(f b)$ \\
\hline$[\mathrm{I}-8]$ & 439 & 4.387 \\
\hline [II-9] & 379 & 6.648 \\
\hline \multirow{2}{*}{$\begin{array}{l}\text { SubPrs. } \\
\text { NO. }\end{array}$} & \multicolumn{2}{|c|}{$\tan \beta=30$} \\
\hline & $\sqrt{\mathrm{s}}(\mathrm{GeV})$ & $\sigma(f b)$ \\
\hline [I-8] & 457 & 3.778 \\
\hline [II-9] & 418 & 5.750 \\
\hline
\end{tabular}

\section{References}

1- C. Csaki, “The Minimal Supersymmetric Standard Model (MSSM),” Mod.Phys.Lett., vol. A11, 1996, p. 599.

2- D.I. Kazakov, "Beyond the Standard Model,” hepph, Caramulo, Portugal: 2000.

3- P. Roy, "Mechanisms of Supersymmetry Breaking in the MSSM," Pramana 60, Harish-Chandra Research Institute, 2002.

4- J. Ellis, "Decline and Fall of the Standard Model," CERN-TH/2001-275, 2001.

5- P. Langacker, "Structure of the Standard Model," Precision Tests of the Standard Electroweak Model, ed. P. Langacker (World, Singapore, 1995), 2003, p. 15.

6- S.F. Novaes, "Standard Model- An Introduction," hep-ph, (World Scientific, Singapore): 2000.

7- A.C.F.A. Group, Particle Physics Experiments at JLC, ACFA Linear Collider Working Group, 2001.

8- S. Heinemeyer and others, Cern-th/2000-369, 2000.

9- E. Accomando and others, "Physics Reprt 299," 1998, pp. 1-78.

10- P. 3 TESLA Technical Design Report, "Physics at an e+ e- Linear Collider," hep-ph/0106315.

11- J. Ellis and others, "On the Higgs mass in the CMSSM,” Phys. Letter B633, 2006, pp. 583-590.

12- L.E.W. Group, "status of August 2005."

13- O. collaborations DELPHI L3 The ALEPH and L.H.W. Group, hep-ex/0602042, 2006.

14- T.O. Collaboration, "Search for Neutral Higgs Bosons in CP-Conserving and CP-Violating MSSM Scenarios,” 2004.
15- J.A. Aguilar-Saavedra and others, Physics at an e+ e- Linear Collider, 2001.

16- A. DJOUADI, "The Search for Higgs particles at high energy colliders: Past, Present and Future," Review talk given at the Workshop on High Energy Physics Phenomenology,WHEPP VII, HarishChandra Research Institute, Allahabad, India: 2002.

17- T.A.E.-A. Ibrahim S. Mahmoud, "Production of Lightest Neutral MSSM Higgs Boson in Association with s-Lepton Pairs at Electron Positron Colliders,” International Review of Physics (IREPHY), vol. 6, 2012, pp. 488-493.

18- S.P.Martin, http://zippy.physics.niu.edu/modellines.html.

19- S.P. Martin, S. Moretti, J. Qian, and G.W. Wilson, "Snowmass," pp. 3-46.

20- D. Groom and others, Eur. Phys. J., vol. C 15, 2000, p. 1.

21- A. Sopczak, "MSSM Higgs Boson Searches at LEP," The 13th International Conference on Supersymmetry and Unification of Fundamental Interactions (SUSY 05), Durham, UK: 2005.

22- T.L. Collaborations, "Search for Neutral MSSM Higgs Bosons at LEP,” Eur.Phys.Journal, vol. C, 2007.

23- M. Carena, S. Heinemeyer, C. Wagner, and G. Weiglein, .

24- A. Pukhov, CalcHEP - Calculation for high energy physics -a package for evaluation of Feynman diagrams, integration over multi-particle phase space, and event generation, User's manual for version 2.1, 2002. 